\title{
High-resolution 3D imaging of integrated circuits by X-ray ptychography
}

\author{
Michal Odstrcil, Mirko Holler, Jorg Raabe, Manuel Guizar-Sicairos \\ Paul Scherrer Institut, 5232 Villigen PSI, Switzerland
}

\begin{abstract}
Significant advances in lithography and chip manufacturing in recent years have resulted in new challenges in metrology of electronic microdevices. Manufacturing process for the $10 \mathrm{~nm}$ node is already available and in combination with complex three-dimensional structured interconnections, there is a lack of methods for verification that the final products correspond to the original specifications. X-ray ptycho-tomography is a locally nondestructive imaging method that could potentially help to fill the gap between electron microscopy and conventional X-ray tomography. Quantitativeness of ptycho-tomography provides detailed device geometries and corresponding sensitivity to elemental composition through the complex-valued refractive index. In order to tackle the experimental challenges and improve the imaging quality, new computational methods need to be developed for both ptychography and tomography that can account for a wide range of imperfections such as sample drifts, illumination changes or sample changes during the scan. Here, we will mention the most important of them and possible ways how to deal with them.
\end{abstract}

Keywords: X-ray, ptychography, coherent diffractive imaging, tomography

\section{INTRODUCTION}

Inspection of printed integrated circuits (IC) is a challenging but important task with various applications such as verification if the delivered IC satisfies required specifications, detection of potential security threats or verifying intellectual property of potentially cloned IC. In particular, hardware security is of emerging importance due to the fact that chip production is often outsourced to multiple external manufactures and without appropriate tools for verification of the delivered products, the IC can contain unapproved modifications or vulnerabilities.

The traditional metrology methods, such as destructive methods based on delayering of IC layers and imaging by scanning electron microscopy ${ }^{1}$, are close to their limits already for the current lithography processes and additionally delayering of and imaging a large chip is a time-consuming and destructive process, where important features of the IC can be lost. One of the methods that have the potential to alleviate these issues is high-resolution X-ray ptychographic microscopy. Due to high penetration of the X-ray light, it is currently possible to image interior structures of the IC at $15 \mathrm{~nm}$ isotropic 3D resolution ${ }^{2}$ with future outlook to sub-10 $\mathrm{nm}$ resolution. Here, we will discuss some of the potential directions for the future development and current challenges that need to be tackled to reach reliable sub-10 nm resolution.

In the last decade, X-ray microscopy has been undergoing rapid development because of the advent of coherent diffractive imaging (CDI) methods, also called lensless imaging methods. These make possible to image samples at resolutions far beyond limits of the traditional lens based X-ray full-field microscopy. Imaging using X-rays has many benefits compared to other methods. The two main advantages are the potential to image nanometer scale features due to the short wavelength light used for imaging, usually from 0.5 to $0.1 \mathrm{~nm}$, and also the good penetration of high-energy Xrays through most materials. Additionally, thanks to the flexibility of synchrotron-based X-ray sources, the X-ray energy can be tuned to match a given application, i.e. optimize penetration and contrast. For example X-ray radiation around $\sim 6 \mathrm{keV}$ has good penetration through most of materials and therefore, it can be used to image interior of samples with tens of microns thickness. Good penetration and long depth of focus makes X-ray microscopy a promising approach for nanotomography. On the other hand, good penetration of X-rays leads to low absorption contrast. This is the reason, why the phase-contrast which is produced by additional phase-shift of the illumination wavefront after passing the object, is preferred for high-resolution X-ray imaging.

Image Sensing Technologies: Materials, Devices, Systems, and Applications V, edited by

Nibir K. Dhar, Achyut K. Dutta, Proc. of SPIE Vol. 10656, 106560U · C 2018 SPIE

CCC code: $0277-786 \mathrm{X} / 18 / \$ 18 \cdot$ doi: $10.1117 / 12.2304835$ 
In order to benefit from the phase-contrast and avoid the need of high-resolution X-ray optics, we have used a scanningbased CDI method called ptychography ${ }^{3,4}$. During ptychographic imaging, the sample is scanned through a coherent structured illumination probe and the scattered signal is collected downstream of the sample. Since only intensity of the scattered light can be directly measured, the phase of the incident photons is lost and is retrieved using additional constraints. For ptychography, the additional constraints are given by the overlap between the adjacent illuminated regions. Sufficient overlap provides enough information to reconstruct attenuation and relative phase-shift caused by the sample, as well as the unknown complex-valued structured illumination probe. Also, since the size of each illuminated region and the scanning step size are orders of magnitude larger than the achieved resolution, ptychography is able to provide high imaging speed ${ }^{5}$ compared to standard scanning transmission X-ray microscopy (STXM). Ptychography is able to separate the illumination wavefield from the reconstructed object phase-shift and it provides the complex-valued sample transmission function. In combination with tomography, ptychographic X-ray computed tomography (PXCT), provides the local electron density of the sample. This can help to identify the material distribution inside the samples ${ }^{6}$. PXCT can nowadays reliably reach sub-20 nm isotropic 3D resolution for highly scattering samples ${ }^{2,7}$ and therefore PXCT has broad applications in material ${ }^{8,9}$ and biological sciences ${ }^{10-13}$. Additionally, with the emerging diffraction limited X-ray sources that promise increased coherent flux by several orders of magnitude ${ }^{14,15}$, PXCT has the potential to be used for large-scale imaging tasks, such as the mentioned IC inspection as show in Fig 1.
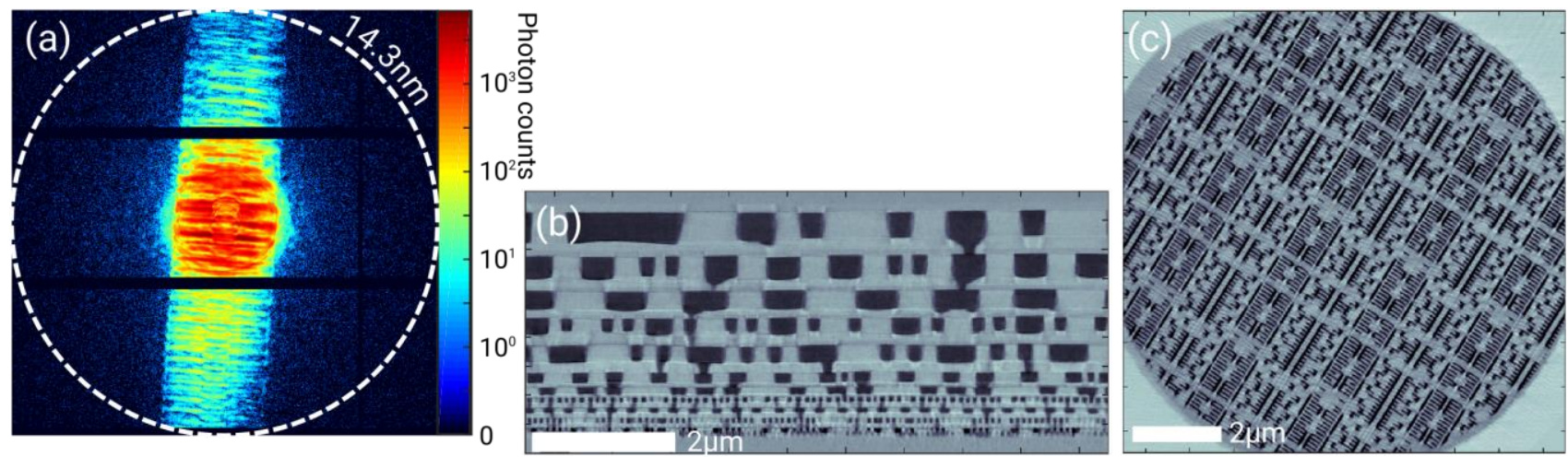

Figure 1 (a) Example of measured diffraction pattern for $100 \mathrm{~ms}$ exposure time at $6.2 \mathrm{keV}$ for an Intel chip produced by $22 \mathrm{~nm}$ technology ${ }^{2}$ measured at the cSAXS beamline, Paul Scherrer Institut, Switzerland. $(b, c)$ Tomographic cuts from the PXCT reconstruction of electron density of the same sample.

\section{CHALLENGES OF HIGH RESOLUTION IC IMAGING BY PTYCHOGRAPHY}

High resolution imaging of IC by PXCT method is a highly demanding task, because high resolution with large field of view are important for this application. Ptychography theoretically enables reaching high resolution limited only by the largest collected diffraction angles with sufficient signal to noise ratio (SNR), however similarly to other CDI methods, ptychography is based on multiple assumptions whose limited validity may lead to deterioration of the reconstruction quality. The standard assumptions are:

a) Precise knowledge of the relative positions of the sample and the illumination probe

b) Constant illumination, both long term, i.e. over duration of single scan and short term, i.e. stability during a single exposure.

c) Sample is sufficiently thin so that exit-wave $\Psi_{i}(x)$ can be described as a product

$$
\Psi_{i}(x)=P(x) O\left(x-x_{i}\right)
$$

between $P(x)$, the complex-valued illumination probe, and $\mathrm{O}(x)$, the complex-valued transmissivity of the imaged object.

d) The measured diffraction pattern is sufficiently sampled 
e) Illumination is fully coherent

f) The measured intensity can be well described by the following expression

$$
I=|F\{\psi\}|^{2},
$$

where $F$ denotes a a propagator between the sample and detector.

g) Sample is static, neither moving nor changing, during the scan

h) Noise in data complies well with the statistical model of the reconstruction method ${ }^{16,17}$

i) The illumination probe is sufficiently structured ${ }^{18}$

j) Absence of incoherent background signal, e.g. caused by elastic scattering by air or windows ${ }^{16,19,20}$ or read-out noise in data ${ }^{21}$

Real experiments can only approach these assumptions, although often closely enough so that other factors become limiting such as low contrast of the images sample. However, since low contrast seems not to be the major limitation for chip imaging, as it can be seen in Fig 1a, where intensities with high signal-to-noise ratio are shown beyond scattering angles corresponding to $14.3 \mathrm{~nm}$, the ultimate achievable resolution is likely limited by a combination of some of the assumptions outlined above.

One of the major advantages of ptychography is its flexibility, because it allows computationally correcting for many of the mentioned experimental limitations. Some of them can be corrected without loss of quality while others, e.g. incohererent illumination or incoherent background, entail loss of information and will unavoidably lead to reduced signal-to-noise ratio.

\subsection{Scanning precision}

If the sample has sufficiently good contrast and structure, i.e. has features on a broad range of spatial frequencies, ptychography allows to estimate positions of the illumination probe with subpixel precision ${ }^{16,22,23}$. This is a critical feature for verification of scanning accuracy of the ptychography setups ${ }^{24}$, where precision of the interferometry system is often far better than the required resolution ${ }^{25}$, however the position errors can be dominated by systematic errors and therefore the overall accuracy of the positioning system can still be a limiting factor. An example in Fig. 2 shows the result of applying position refinement ${ }^{16}$ with a scan from the flOMNI instrument ${ }^{7}$ at the cSAXS beamline, Paul Scherrer Institut, Switzerland. The scan was performed at $6.2 \mathrm{keV}$ energy with half-period resolution $38 \mathrm{~nm}$. The arrows show the direction of position correction with its magnitude 10 times exaggerated for visualization. One can clearly see high local correlation between the errors and the height of the points on the scan that are arranged in horizontal slabs of approximately $300 \mu \mathrm{m}$ period. Standard deviation of the short distance horizontal errors, i.e. excluding the long range linear drift of the position, is below $10 \mathrm{~nm}$ and below $5 \mathrm{~nm}$ in the horizontal and vertical directions, respectively. Note that the resolution of the current state of art X-ray nanotomography, with this instrument, is around $15 \mathrm{~nm}^{2}$, while for the latter the current performance is adequate it needs to be improved for sub-10 $\mathrm{nm} 3 \mathrm{D}$ imaging.
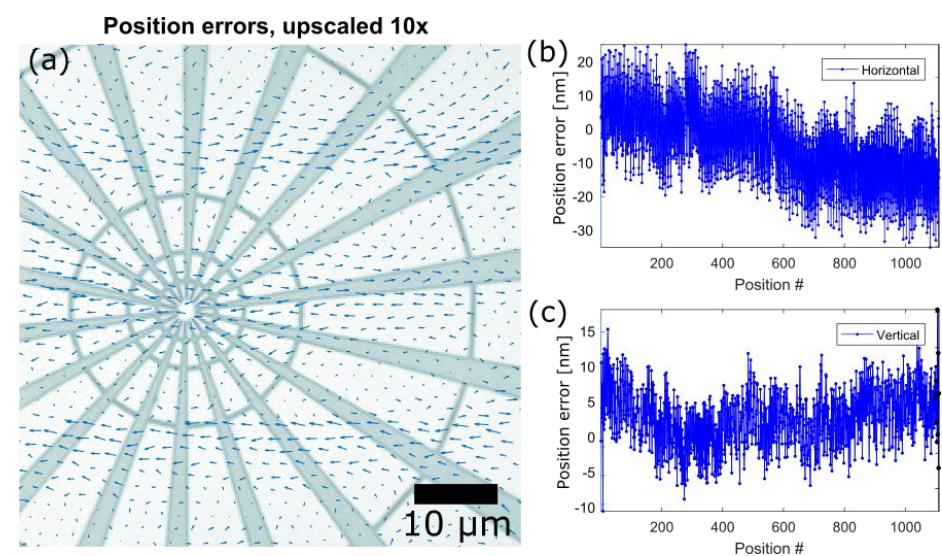

Figure 2: Estimation of position errors for the fLOMNI instrument ${ }^{7}$ for a test sample ${ }^{7}$. (a) Vector map of the position errors overlapped with the reconstructed test object. Arrows are showing direction of the update with $10 \times$ increased magnitude for visualization. Plots $(b, c)$ shows residuum between the original and refined positions for horizontal $(b)$ and vertical (c) directions. 
An issue closely related to the position accuracy is the vibrations of the imaging setup. Vibrations of the illumination probe with respect to the object that are faster than the exposure time will unavoidably cause smearing of the measured diffraction pattern. The effect of vibration much faster than the exposure time is similar to that of partial coherence that in some cases can be accounted for by mixed-modes reconstructions ${ }^{26,27}$ or correction of the propagated wavefront $\operatorname{model}^{28}$, however it will unavoidably lead to lower photon efficiency, i.e. higher imaging dose is needed to reach similar resolution ${ }^{29-31}$. Therefore, the vibration amplitude should be significantly below the targeted resolution. Histograms of the position precision of the fLOMNI instrument ${ }^{7}$ shown in Fig. 3 indicate that the average stability of the setup is around $9 \mathrm{~nm}$ in the horizontal direction and around $7 \mathrm{~nm}$ in the vertical direction.

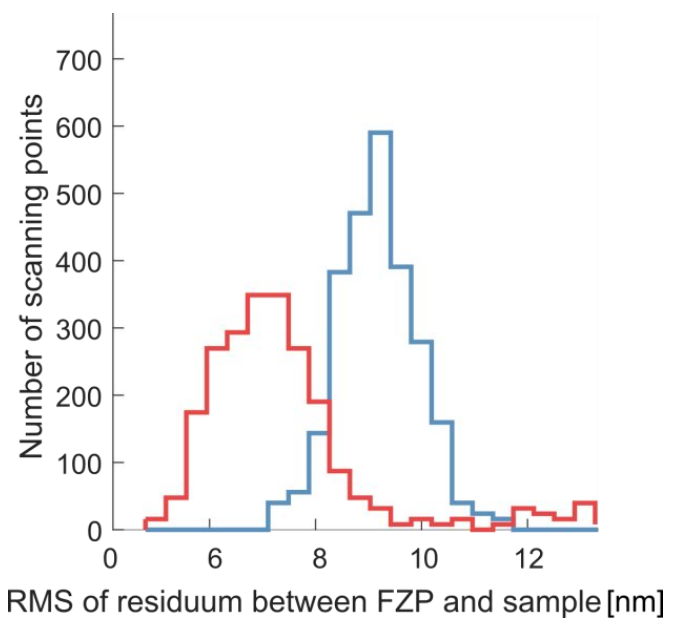

Figure 3. Horizontal (blue) and vertical (red) level of vibrations between sample and the illumination optics indicated by standard deviation of the position during 1600 exposures taken by the fLOMNI instrument ${ }^{7}$.

Sufficient coherence of the illumination, short term illumination stability and sufficient sampling of the measured patterns are also crucial requirements of ptychography ${ }^{32,33}$ and in spite of ptychography offers method to deal with these effects $^{26,27,34,35}$, if these conditions are not met, reconstruction quality will be degraded for a given dose. In particular partially coherent illumination may seem to be an easy way to increase the flux and thus to reduce the exposure time needed to reach a target resolution ${ }^{36,37}$. However, low coherence, similarly to setup vibrations or insufficient diffraction sampling, leads to loss of speckle visibility. Therefore the actual quality improvement provided by the additional flux of a low coherence illumination could be smaller than the expected value or even counterproductive ${ }^{38}$. On the other hand, one should not use more coherence than needed ${ }^{32}$ as this leads to unnecessary filtering of the incoming intensity and decrease of the signal-to-noise ratio, an optimum has to be found.

If the illumination is fully coherent, but the decoherence-like effects are caused by low position or angular stability of the illumination beam, one can try to shorten the exposure per scanning position below the vibration period and use the orthogonal probe relaxation algorithm ${ }^{16,39}$ to compensate for the variable illumination. If the exposure is sufficiently shorter than period of the beam vibrations, no information is lost and the photon efficiency of the ptychography method can be preserved. If this is not possible, then the modes-mixture method ${ }^{26}$ can help to reduce the reconstruction blur but always at the price of increased imaging dose. A similar effect can be also seen for fly-scan ptychography ${ }^{29-31}$, when the sample is moved continuously during the exposure. In that case, an increased length of the scanning step leads to a significant increase of the photon dose required to maintain the imaging quality ${ }^{40,41}$

\subsection{Depth of focus limit}

Another limitation that is in particular related to high-resolution X-ray ptychography is depth of focus (DoF). DoF scales quadratically with the resolution $\delta x$ with dependence estimated for ptychography ${ }^{42}$ as 


$$
\text { DoF }=5.2 \frac{\delta x^{2}}{\lambda}
$$

where $\lambda$ is the imaging wavelength. Therefore, the DoF is a serious limitation when the resolution is approaching the imaging wavelength. For example, to reach a resolution $10 \mathrm{~nm}$ using $6.2 \mathrm{keV}$ photon energy, the sample should be smaller than $2.5 \mu \mathrm{m}$. This can be slightly relaxed by moving to higher energy illumination; however this will often reduce the sample contrast and the available coherent flux, thus ultimately lowering the image quality. Another option is to increase the scanning overlap in ptychography and reduce exposure per position, as this leads to a more relaxed $\mathrm{DoF}^{42}$. However, versatility of the ptychography algorithms allows to relax our assumption in Eq. 1 and rather describe the object by multiple layers separated by free space ${ }^{42-45}$. Although this method can significantly extend the limits of the DoF, compared to standard ptychography the multilayer ptychography methods have significant increased computational time per iteration, slower convergence, and it requires smaller scanning step in order to sufficiently constraint the reconstruction of multiple layers. Limited DoF can be one of the explanations why projection collected at 0 degrees is not precisely matching a projection collected at 180 degrees, as shown in Fig. 3. This problem is exacerbated when aiming for sub- $10 \mathrm{~nm}$ resolution ${ }^{46}$.
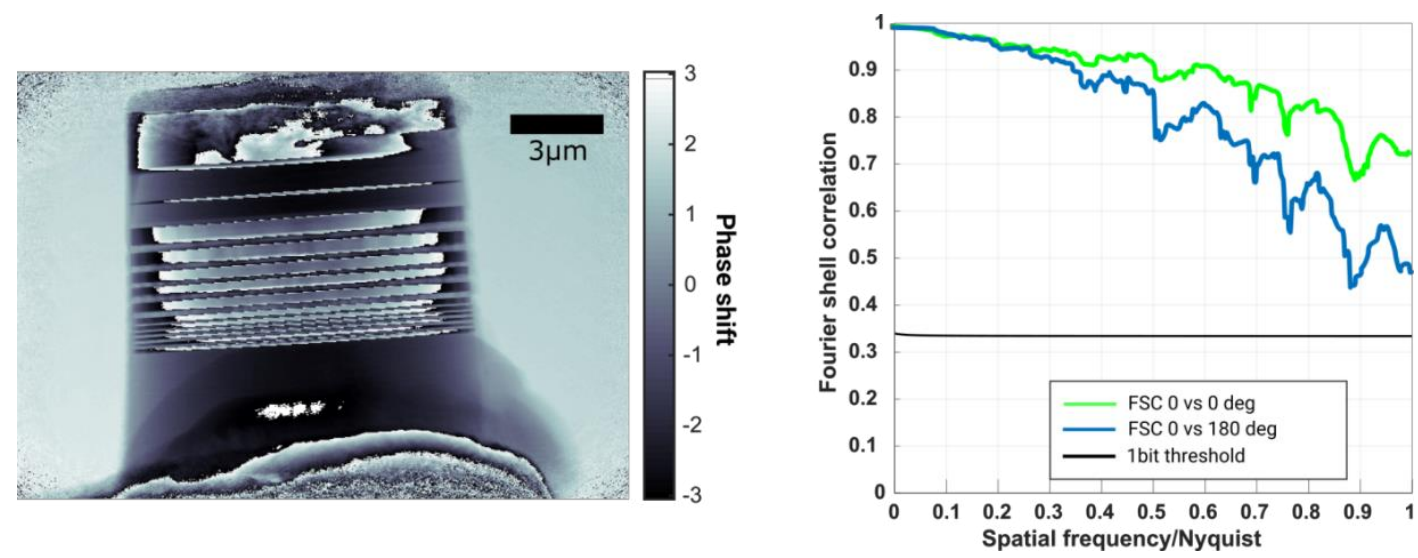

Figure 4. (a) Phase projection of IC sample measured at the cSAXS beamline ${ }^{2}$, at 0 degrees before phase unwrapping. (b) Fourier ring correlation (FRC) between two projections acquired at 0 degrees compared with FRC between one projection at 0 and 180 degrees. The projections were aligned, phase-ramp was removed and the projection at 180 was horizontally flipped. The pixel size corresponding to the Nyquist frequency equal 1 is $14.3 \mathrm{~nm}$.

\subsection{Phase gradients}

Another constraint that limits the maximum thickness of the measured IC is phase unwrapping, which is needed for PXCT. Our current approach ${ }^{47}$ avoids direct unwrapping of data by using the phase derivative of the complex-valued projections as an input to a modified filtered back-projection (FBP) method. An assumption of this method is however that the phase change between two pixels is smaller than $\pi$. This condition results in artifacts in the IC reconstructions because they contain many straight, sharp and high contrast features, as can be seen in Fig 4 . This constraint is not truly a limitation of maximal achievable resolution, however large phase gradients will rather lead to artifacts close to the sharp features and it makes mutual alignment of the projections more difficult. This effect can be reduced if resolution of the reconstruction is further increased so that the phase change per pixel is smaller than $\pi$. The latter limits PXCT to relatively small IC samples in order of tens of microns in diameter, at least in the standard tomography geometry.

\subsection{Static sample}

Finally, the imaged sample has to be static during the entire PXCT scan. Rigid movements between the PXCT projections caused for example by slow thermal drifts can be accounted for by a self-consistent alignment method ${ }^{48,49}$. Sample changes that cannot be described by rigid move may lead to quality deterioration and ideally one should use cryo-ptychographic instruments to at least partly mitigate the radiation damage ${ }^{24,50}$. 


\section{CONCLUSION AND OUTLOOK}

Many of the mentioned challenges can be removed by optimization and further development of PXCT instrument, Due to the planar geometry of the IC die with the active area up to $1 \mathrm{~cm}^{2}$ and thickness of the region of interest in order of ten microns a laminography configuration is better suited for this task. For this it is necessary to use more general algorithms. Similarly to PXCT method, Ptychographic X-ray laminography ${ }^{2}$ (PyXL) estimates interior density of the sample from complex-valued projections obtained by ptychography. Unlike in tomography, the rotation axis in laminography is not perpendicular to the propagation direction of the illumination beam, as indicated in Fig 6. The total field of view of PyXL is limited only by the scanning range and the sample thickness, related to the DoF, is only proportional to the distance between the first and the last IC layer and the secant of the laminography angle. Additionally the maximum phase gradient is more likely to be small because IC interconnections are generally not parallel to the illumination beam.

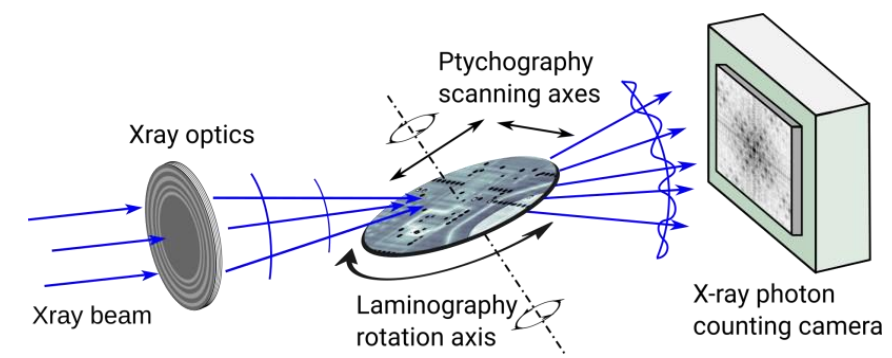

Figure 5. Illustration of the ptychographic X-ray laminography imaging setup.

A consequence of the tilted rotation axis is that for laminography the Fourier space of the reconstructed volume is not fully angularly covered. Therefore, the reconstruction will contain artifacts unless additional priors are used. This is shown in Fig. 7b, were the measured PXCT reconstruction of a Pentium chip $^{2}$ (Fig 7d) is compared to a simulated reconstruction of the same sample in the laminography configuration with 60 degrees angle between the illumination and the rotation axis as indicated in Fig 7a. The loss of quality can be partly reversed by using simple additional priors as shown in Fig 7c. Further improvements may be possible by including knowledge of the manufacturing process into the reconstruction as well.

(a)

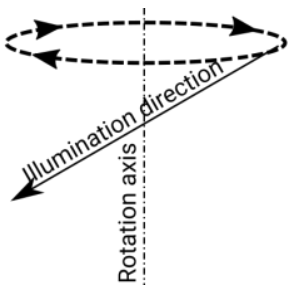

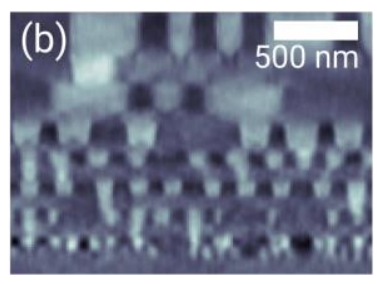

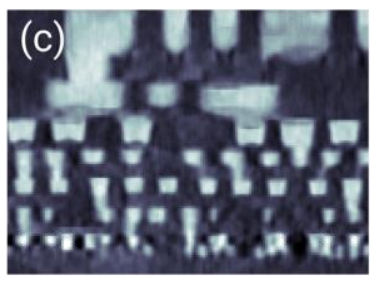

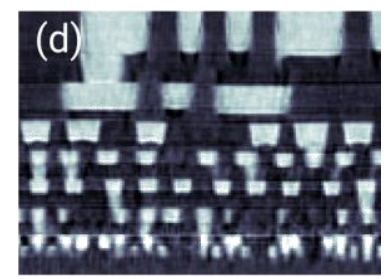

Figure 6: (a) Laminography geometry used for simulation of the laminography reconstruction (b). (c) Laminography reconstruction after introduction of basic priors and (d) is original PXCT reconstruction that was used as phantom for (b) and (c).

\section{REFERENCES}

[1] Torrance, R. and James, D., "The state-of-the-art in semiconductor reverse engineering," Proc. 48th Des. Autom. Conf. - DAC '11, 333 (2011).

[2] Holler, M., Guizar-Sicairos, M., Tsai, E. H. R. R., Dinapoli, R., Müller, E., Bunk, O., Raabe, J. and Aeppli, G., "High-resolution non-destructive three-dimensional imaging of integrated circuits," Nature 543(7645), 402-406 (2017).

[3] Rodenburg, J. M. and Faulkner, H. M. L. L., "A phase retrieval algorithm for shifting illumination,” Appl. Phys. Lett. 85(20), 4795-4797 (2004). 
[4] Rodenburg, J. M., Hurst, A. C., Cullis, A. G., Dobson, B. R., Pfeiffer, F., Bunk, O., David, C., Jefimovs, K. and Johnson, I., "Hard-X-ray lensless imaging of extended objects," Phys. Rev. Lett. 98(3), 1-4 (2007).

[5] Guizar-Sicairos, M., Johnson, I., Diaz, A., Holler, M., Karvinen, P., Stadler, H.-C., Dinapoli, R., Bunk, O. and Menzel, A., "High-throughput ptychography using Eiger: scanning X-ray nano-imaging of extended regions," Opt. Express 22(12), 14859-14870 (2014).

[6] Diaz, A., Trtik, P., Guizar-Sicairos, M., Menzel, A., Thibault, P. and Bunk, O., "Quantitative X-ray phase nanotomography," Phys. Rev. B 85(2), 20104 (2012).

[7] Holler, M., Diaz, A., Guizar-Sicairos, M., Karvinen, P., Färm, E., Härkönen, E., Ritala, M., Menzel, A., Raabe, J. and Bunk, O., "X-ray ptychographic computed tomography at $16 \mathrm{~nm}$ isotropic 3D resolution," Sci. Rep. 4 (2014).

[8] Esmaeili, M., Fløystad, J. B., Diaz, A., Høydalsvik, K., Guizar-Sicairos, M., Andreasen, J. W. and Breiby, D. W., "Ptychographic X-ray tomography of silk fiber hydration," Macromolecules 46(2), 434-439 (2013).

[9] Birkbak, M. E., Guizar-Sicairos, M., Holler, M. and Birkedal, H., "Internal structure of sponge glass fiber revealed by ptychographic nanotomography,” J. Struct. Biol. 194(1), 124-128 (2016).

[10] Dierolf, M., Menzel, A., Thibault, P., Schneider, P., Kewish, C. M., Wepf, R., Bunk, O. and Pfeiffer, F., "Ptychographic X-ray computed tomography at the nanoscale," Nature 467(7314), 436-439 (2010).

[11] Wilts, B. D., Sheng, X., Holler, M., Diaz, A., Guizar-Sicairos, M., Raabe, J., Hoppe, R., Liu, S. H., Langford, R., Onelli, O. D., Chen, D., Torquato, S., Steiner, U., Schroer, C. G., Vignolini, S. and Sepe, A., "EvolutionaryOptimized Photonic Network Structure in White Beetle Wing Scales," Adv. Mater. (2017).

[12] Zanette, I., Enders, B., Dierolf, M., Thibault, P., Gradl, R., Diaz, A., Guizar-Sicairos, M., Menzel, A., Pfeiffer, F. and Zaslansky, P., "Ptychographic X-ray nanotomography quantifies mineral distributions in human dentine," Sci. Rep. 5 (2015).

[13] Deng, J., Vine, D. J. D. D. J., Chen, S., Nashed, Y. S. G., Jin, Q., Phillips, N. W., Peterka, T., Ross, R., Vogt, S. and Jacobsen, C. J., "Simultaneous cryo X-ray ptychographic and fluorescence microscopy of green algae," Proc. Natl. Acad. Sci. 112(8), 2314-2319 (2015).

[14] Hettel, R., "DLSR design and plans: An international overview," J. Synchrotron Radiat. 21(5), 843-855 (2014).

[15] Thibault, P., Guizar-Sicairos, M. and Menzel, A., "Coherent imaging at the diffraction limit," J. Synchrotron Radiat. 21(5), 1011-1018 (2014).

[16] Odstrčil, M., Menzel, A. and Guizar-Sicairos, M., "Iterative least-squares solver for generalized maximumlikelihood ptychography," Opt. Express 26(3), 3108 (2018).

[17] Thibault, P. and Guizar-Sicairos, M., "Maximum-likelihood refinement for coherent diffractive imaging," New J. Phys. 14(6), 63004 (2012).

[18] Guizar-Sicairos, M., Holler, M., Diaz, A., Vila-Comamala, J., Bunk, O. and Menzel, A., "Role of the illumination spatial-frequency spectrum for ptychography," Phys. Rev. B - Condens. Matter Mater. Phys. 86(10), 1-4 (2012).

[19] Marchesini, S., Schirotzek, A., Yang, C., Wu, H. T. and Maia, F., “Augmented projections for ptychographic imaging," Inverse Probl. 29(11) (2013).

[20] Reinhardt, J., Hoppe, R., Hofmann, G., Damsgaard, C. D., Patommel, J., Baumbach, C., Baier, S., Rochet, A., Grunwaldt, J. D., Falkenberg, G. and Schroer, C. G., "Beamstop-based low-background ptychography to image weakly scattering objects," Ultramicroscopy 173(October 2016), 52-57 (2017).

[21] Ang, C. H. W., Ijian, Z. X. U., Iu, H. A. L., Ang, Y. O. N. G. W., Ang, J. I. A. N. W., Ai, R. E. T., Wang, C., Xu, Z., Liu, H., Wang, Y., Wang, J. and Tai, R., "Background noise removal in X-ray ptychography," Appl. Opt. 56(8), 2099 (2017).

[22] Zhang, F., Peterson, I., Vila-Comamala, J., Diaz, A., Berenguer, F., Bean, R., Chen, B., Menzel, A., Robinson, I. K. and Rodenburg, J. M., "Translation position determination in ptychographic coherent diffraction imaging," Opt. Express 21(11), 13592 (2013).

[23] Guizar-Sicairos, M. and Fienup, J. R., "Phase retrieval with transverse translation diversity: a nonlinear optimization approach.," Opt. Express 16(10), 7264-7278 (2008).

[24] Holler, M., Raabe, J., Diaz, A., Wepf, R., Odstrcil, M., Shaik, F. R., Panneels, V., Menzel, A., Sarafimov, B., Maag, S., Wang, X., Thominet, V., Walther, H., Lachat, T., Vitins, M. and Bunk, O., “OMNY - a tOMography Nano crYo stage," Res. Sci. Instruments submitted (2018).

[25] Holler, M. and Raabe, J., "Error motion compensating tracking interferometer for the position measurement of objects with rotational degree of freedom," Opt. Eng. 54(5), 54101 (2015). 
[26] Thibault, P. and Menzel, A., "Reconstructing state mixtures from diffraction measurements.," Nature 494(7435), 68-71 (2013).

[27] Clark, J. N. and Peele, A. G., "Simultaneous sample and spatial coherence characterisation using diffractive imaging," Appl. Phys. Lett. 99(15), 154103 (2011).

[28] Burdet, N., Shi, X., Parks, D., Clark, J. N., Huang, X., Kevan, S. D. and Robinson, I. K., "Evaluation of partial coherence correction in X-ray ptychography," Opt. Express 23(5), 5452-5467 (2015).

[29] Clark, J. N., Huang, X., Harder, R. J. and Robinson, I. K., "Continuous scanning mode for ptychography.," Opt. Lett. 39(20), 6066-6069 (2014).

[30] Deng, J., Nashed, Y. S. G., Chen, S., Phillips, N. W., Peterka, T., Ross, R., Vogt, S., Jacobsen, C. and Vine, D. J., "Continuous motion scan ptychography: characterization for increased speed in coherent X-ray imaging," Opt. Express 23(5), 5438-5451 (2015).

[31] Pelz, P. M., Guizar-Sicairos, M., Thibault, P., Johnson, I., Holler, M. and Menzel, A., "On-the-fly scans for Xray ptychography," Appl. Phys. Lett. 105(25), 251101 (2014).

[32] Spence, J. C. H., Weierstall, U. and Howells, M., "Coherence and sampling requirements for diffractive imaging.," Ultramicroscopy 101(2-4), 149-152 (2004).

[33] da Silva, J. C. and Menzel, A., "Elementary signals in ptychography," Opt. Express 23(26), 33812-33821 (2015).

[34] Clark, J. N., Huang, X., Harder, R. J. and Robinson, I. K., "Dynamic Imaging Using Ptychography,” Phys. Rev. Lett. 112(11), 113901 (2014).

[35] Edo, T. B., Batey, D. J., Maiden, a. M., Rau, C., Wagner, U., Pešić, Z. D., Waigh, T. a. and Rodenburg, J. M., "Sampling in x-ray ptychography," Phys. Rev. A 87(5), 53850 (2013).

[36] Enders, B., Dierolf, M., Cloetens, P., Stockmar, M., Pfeiffer, F. and Thibault, P., "Ptychography with broadbandwidth radiation,” Appl. Phys. Lett. 104(17), 171104 (2014).

[37] Baksh, P. D., Odstrčil, M., Kim, H.-S., Boden, S. A., Frey, J. G. and Brocklesby, W. S., "Wide-field broadband extreme ultraviolet transmission ptychography using a high-harmonic source,” Opt. Lett. 41(7), 1317 (2016).

[38] Stachnik, K., Mohacsi, I., Vartiainen, I., Stuebe, N., Meyer, J., Warmer, M., David, C. and Meents, A., "Influence of finite spatial coherence on ptychographic reconstruction," Appl. Phys. Lett. 107(1), 11105 (2015).

[39] Odstrcil, M., Baksh, P., Boden, S. A., Card, R., Chad, J. E., Frey, J. G. and Brocklesby, W. S., "Ptychographic coherent diffractive imaging with orthogonal probe relaxation," Opt. Express 24(8), 8360 (2016).

[40] Odstrcil, M., Diaz, A., Holer, M. and Guizar-Sicairos, M., "Path toward fast, high-resolution and more photonefficient X-ray ptychography," Opt. InfoBase Conf. Pap. Part F46-C (2017).

[41] Odstrcil, M., Holler, M. and Guizar-Sicairos, M., “Arbitrary-path fly-scan ptychography," Submitt. to Opt. Express (2018).

[42] Tsai, E. H. R., Usov, I., Diaz, A., Menzel, A. and Guizar-Sicairos, M., "X-ray ptychography with extended depth of field," Opt. Express 24(25), 29089 (2016).

[43] Li, P. and Maiden, A., "Multi-slice ptychographic tomography," Sci. Rep., 1-10 (2018).

[44] Humphry, M. J., Maiden, A. M., Rodenburg, J. M., Humphry, M. J. and Rodenburg, J. M., "Ptychographic transmission microscopy in three dimensions using a multi-slice approach,” J. Opt. Soc. Am. A 29(8), 1606 (2012).

[45] Shimomura, K., Hirose, M. and Takahashi, Y., "Multislice imaging of integrated circuits by precession X-ray ptychography," Acta Crystallogr. Sect. A Found. Adv. 74(1), 66-70 (2018).

[46] Deng, J., Hong, Y. P., Chen, S., Nashed, Y. S. G., Peterka, T., Levi, A. J. F., Damoulakis, J., Saha, S., Eiles, T. and Jacobsen, C., "Nanoscale x-ray imaging of circuit features without wafer etching," Phys. Rev. B 95(10) (2017).

[47] Guizar-Sicairos, M., Diaz, A., Holler, M., Lucas, M. S., Menzel, A., Wepf, R. A. and Bunk, O., "Phase tomography from x-ray coherent diffractive imaging projections," Opt. Express 19(22), 21345 (2011).

[48] Dengler, J., "A multi-resolution approach to the 3D reconstruction from an electron microscope tilt series solving the alignment problem without gold particles," Ultramicroscopy 30(3), 337-348 (1989).

[49] Ramos, T., Jørgensen, J. S. and Andreasen, J. W., "Automated angular and translational tomographic alignment and application to phase-contrast imaging," J. Opt. Soc. Am. A 34(10), 1830 (2017).

[50] Chen, S., Deng, J., Yuan, Y., Flachenecker, C., Mak, R., Hornberger, B., Jin, Q., Shu, D., Lai, B., Maser, J., Roehrig, C., Paunesku, T., Gleber, S. C., Vine, D. J., Finney, L., Vonosinski, J., Bolbat, M., Spink, I., Chen, Z., et al., "The Bionanoprobe: Hard X-ray fluorescence nanoprobe with cryogenic capabilities," J. Synchrotron Radiat. 21(1), 66-75 (2014). 